\title{
LANDSCHAFT IM BLICK DES VOLKSKUNDLERS
}

Am 29. Juli 1962 verunglückte Dr. Richard Weiß, Professor für Volkskunde an der Universität Zürich im Onsernonetal tödlich. Mit ihm schied ein nicht nur seinem engern Fach verpflichteter bedeutender Forscher aus dem Leben. Er befruchtete durch seine ebenso weite wie gründliche Arbeit zahlreiche Nachbarwissenschaften und nicht zuletzt auch die Geographie. Dies vermag wohl am eindrücklichsten die vorliegende Schrift «Zur Erinnerung an Richard Weiß»zu zeigen, welche drei seiner «Beiträge zur Volkskunde der Schweiz»: «Die Brünig-Napf-Reuß-Linie als Kulturgrenze zwischen Ost- und Westschweiz auf kulturkundlichen Karten», "Alpiner Mensch und alpines Leben in der Krise der Gegenwart» und «Landschaft und Volksart»vereinigt 1 . Ihr geographischer Gehalt soll kurz zu würdigen versucht werden. Dabei interessiert vor allem die Studie «Landschaft und Volksart», die für den Bildband «Der Kanton Zürich in der Sammlung Städte und Landschaften der Schweiz» verfaßt worden war. In ihr betont Weiß gleich anfangs. daß man mit der Stadt Zürich beginnen müsse, wenn man rom Kanton reden wolle. Sie gebe der Landschaft den Namen und die Einheit und sei nicht nur ihre Mitte, sondern auch das Herz. «dessen Pulsschlag einen Kreislauf antreibt, in dem Tausende von Menschen täglich von der Stadt angesogen und wieder ausgestoßen werden. Diese Pendlerströme verstädtern das Land mit ihren Siedlungshäusern, Villen und Bungalows. mit ihren Autos, mit ihren Löhnen und ihrer Freizeit, mit ihren Ansprüchen, mit ihrem Lebensstil.»So betrachtet, werde «das starke Herz der Stadt zum gierig saugenden Polvpen. Der Stadtkanton erscheint als zunehmend verstädterter. nivellierter Kanton.»Es gebe jedoch noch eine Betrachtungsweise, die in der unvermeidlichen Bevölkerungsmischung nicht nur städtische Verwüstung des Bauernlandes, sondern fruchtbare und fördernde Annäherung der Stände und ihrer Lebensformen sehe eine Betrachtungsart, in welcher also die Landschaft durch «Verstädterung» gewinne. Man müsse sich dabei allerdings lösen vom romantischen Idealbild einer Natürlichkeit und Ursprünglichkeit, von welcher der sehnsüchtige Städter träume. «Es kann keine unberührt natürliche und keine rein bäuerliche Landschaft geben in einer technischen Welt, in der die Landwirtschaft selber notwendigerweise zur industriellen Landwirtschaft werden muß. Wenn wir das anerkennen, so sehen wir, was immer noch bleibt. Wir sehen die Wirklichkeit der Landschaft, die trotz städtischem Geist und Ungeist ihr eigenes Gesicht wahrt. Und in dieser Physiognomie suchen wir als Zeichen des Eigenlebens die lebendige Vielfalt der Volksart und die großen Linien bleibender Naturgegebenheiten». Nach dieser «individualgesetzlichen»Charakteristik des Standes Zürich schildert Weiß die Vielgestaltigkeit seiner Landschaft, erst mit dem «Hinweis auf iene unzähligen kleinen und eigenen Welten, die sich zwischen Agasul und Ottenbach, zwischen dem Bachtel und dem Rhein entdecken lassen». dann in den «großen und unwandelbaren Gliederungen», die sich in den vier Rand-und Ecklandschaften»des Ober- und Tnterlandes, des Weinlandes und Knonauer Amtes ausprägen und die ebensosehr aus ihrer naturbestimmten Volkskultur wie aus ihrer kulturbestimmten Naturlandschaft zu verstehen sind. Am Beispiel des Zürcher Oberlandes zeichnet R. Weiß schließlich in unübertreffbarer Eindringlichkeit die engen Bezüge, die zwischen Natur und Volksart spielen. etwa die «merkwürdige Spannung. in welcher der Arbeiterbauer lebt: zwischen, Gwerbli' und Fabrik, zwischen altertümlicher Kleinbauernwirtschaft und den unberechenbaren Konjunkturschwankungen einer Exportindustrie. zwischen karger Bergnatur und trügerischem Geldverdienst, zwischen Alpen und Flachland» oder das scheinbare Paradoxon, daß das von Tobeln zerrissene, nach H. Bernhard schlechthin siedlungsfeindliche Waldland im obern Tößtal in einer Zeit «seine Bevölkerung verdoppelte, während der draußen im fruchtbaren, siedlungsgünstigen, von der Natur in ieder Hinsicht bevorzugten ackerbäuerlichen Mittelland die Bevölkerung stagnierte, zahlenmäßig und auch kulturell». Mit dieser sehr lückenhaft wiedergegebenen Darstellung belegt Weiß ebenso überzeugend wie frappierend, welche Vertiefung die volkskundliche Betrachtungsweise der geographischen bietet, namentlich dann, wenn sie sich wie er gleicherweise nüchtern und gefühlsoffen der Landschaft nähert. In den beiden andern Abhandlungen, von denen «die BrünigNapf-Reuß-Linie» an dieser Stelle $(2,19+7,153-175)$ erstmals abgedruckt wurde. sind die skizzierten Beziehungen vor allem durch Grenzbetrachtungen unterstrichen, wobei die zweite über den «alpinen Menschen»zu erweisen trachtet, daß zwar die Alpen nicht nur das geographische, sondern sozusagen das seelische Rückgrat der Schweiz sind und bleiben, daß man daraus aber nicht notwendigerweise eine «Vergöttlichung» des Älplers ableiten dürfe. M. a..W es ist ein besonderes Anliegen des Forschers immer und immer vor schematischer Generalisierung im Bereich der Zusammenhänge Mensch-Natur zu warnen, zu zeigen, daß sie vielmehr differenzierte einmalige Prozesse sind.

Der Verfasser kam mit R. Weiß in den dreißiger Jahren als Exolorator für den Atlas der Schweizer Volkskunde in freundschaftlichen Kontakt. Ihm waren die Aufnahmen der Gemeinden der Ostschweiz anvertraut. In dieser Eigenschaft hatte er. zusammen mit seiner Frau, die infolge des Krieges den Hauptteil der Befragungen zu bewältigen hatte, des öfteren Gelegenheit, mit R. Weiß Fragen der Beziehungen von Volks- und Landschaftskunde zu erörtern. Dieser hatte schon zu Beginn seiner volkskundlichen Tätigkeit die sogenannte «geographische Methode»als eine wichtige Grundlage der volkskundlichen Forschung nostuliert. wohei sie ihm anfänglich weitgehend mit kartographischer Darstellung identisch schien. Im Zuge jener Gespräche konnte

1 Verlag Eugen Rentsch, Erlenbach-Zürich. 
mit Freude ebenso der Wandel zu einer Auffassung der Geographie als umfassender Landschaftskunde verfolgt werden, wie der Geograph sich mehr und mehr von der Notwendigkeit vermehrten Einbaus volkskundlichen Gedankenguts in die Erfassung der Landschaften überzeugte. In den spätern Werken von Richard Weiß ist die korrelative Schau von der Landschaft zum Volk und vom Volk zur Landschaft zur Meisterschaft entwickelt worden, und die hier besprochene Erinnerungsschrift gibt davon viele packende Beispiele. Mit seinem Biographen, Karl Meuli, der ihr ein ausgezeichnetes Charakterbild des Verstorbenen vorangestellt hat, ziemt auch dem Geographen, der nicht weniger von seinem allzu frühen Scheiden erschüttert war als seine Fachgenossen, Freunde, Verwandte, zu sagen: «Dank für das, was er der Wissenschaft gewesen ist; und es ziemt uns der Wille. ihm Treue zu halten, sein Erbe nach Kräften zu wahren und zu mehren».

E. WINKLER

\section{WALTER KÜMMERLY 60jährig}

Am 9. November dieses Jahres feierte Walter Kümmerly im Kreise seiner Familie und seiner Mitarbeiter den 6o. Geburtstag. Zu diesem Ereignis entbieten ihm die Leser der «Geographica Helvetica» insbesondere aber die Fachgeographen des ganzen Landes, herzlichen Glückwunsch.

Wer kennt nicht den Namen der Firma Kümmerly \& Frey in Bern, deren Leitung Walter Kümmerly zusammen mit Max Frey heute innehat. Längst schon über unsere Landesgrenzen hinaus bekannt für seine Karten, hat der Geographische Verlag Kümmerly \& Frey in den letzten Jahren mehr und mehr auch das Buch in den Vordergrund seiner Erzeugnisse gerückt: Wanderbücher, Reiseliteratur. länderkundliche Werke sind neben prachtvollen Bildbänden entstanden. In weitesten Kreisen ist der Name Kümmerly von der Schulwandkarte der Schweiz her ein vertrauter Begriff. Ihr Schöpfer war der Vater des heutigen Jubilaren. Unentwegt setzt auch Walter Kümmerly seine ganze Kraft für die Kartographie, den Buchverlag und insbesondere für das geographische Lehrmittel ein. Bei allen geschäftlichen Erfolgen und trotz des internationalen Rufes seiner Firma und damit der schweizerischen Kartographie bleibt er im persönlichen Umgang stets derselbe bescheidene und hilfsbereite Mensch, als den ihn vor allem auch die Berner Geographen kennen. Seit vielen Jahren ist der Jubilar Vorstandsmitglied der Geographischen Gesellschaft Bern, wo sein sachliches Urteil und seine kollegiale Mitarbeit sehr geschätzt werden. In zahlreichen Fällen hat er unsere Publikationen unterstützt und bereichert, wofür ihm an dieser Stelle einmal öffentlich gedankt sei.

Wer Walter Kümmerly begegnet, würde ihm keine sechs Jahrzehnte ansehen. Mögen ihm Spannkraft und Frische noch auf lange hinaus erhalten bleiben und ihn befähigen, weiterhin so zielbewußt und erfolgreich zu arbeiten!

w. KUHN

\section{REZENSIONEN - COMPTES-RENDUS CRITIQUES}

Geotechnische Karte der Schweiz 1:2000oo. Zweite Auflage. Bern 1963. Kümmerly \& Frey. Gefalzt.

Die erste Auflage dieser Karte erschien 1934-1938; sie war rasch vergriffen, da sie nicht nur für Geologen, sondern auch für Praktiker des Bauwesens von großem Interesse war. Die geotechnische Kommission beschloß deshalb 1956, die Karte neu herauszugeben, wobei eine eingehende Überarbeitung als Selbstverständlichkeit erschien. Als erstes Blatt ist die Nordostkarte Luzern-Zürich-St. Gallen-Chur erhältlich. Die Aufnahmen erfolgten 1957-1962. In die Kartenlegende wurden u. a. die für die Silikose wichtigen Quarzgehalte der ausge. schiedenen Gesteine eingefügt. Eine Nebenkarte 1:1 Mio von H. JäckL bietet zudem eine Übersicht der eiszeitlichen Vergletscherungen. Die weitern Blätter sollen in einjährigen Abständen folgen. Man muß sich die Schwierigkeiten vor Augen halten, die der Erneuerung eines solchen gesamtschweizerischen Kartenwerkes ent- gegenstanden, und wird dann dem Präsidenten der Schweiz. Geotechnischen Kommission, Prof. Dr. F. DE Quervain, und seinen Mitarbeitern umso größere Anerkennung für die ausgezeichnete Leistung ausdrücken, welche schon das erste Blatt repräsentiert. Auch diese zweite Auflage wird zweifellos den Geologen und allen andern Benützern zu einem unentbehrlichen Hilfsmittel werden.

G. SCHUMACHER

Binggeli, Valentin: Der Lukmanier. Schweizer Heimatbücher H. 115. Bern 1963. Paul Haupt. 56 Seiten, 35 Abbildungen. Geheftet Fr. 5.- -

So unbekannt, wie der Verfasser dieses neuen Heimatbuches meint, ist der Lukmanier nun doch nicht; abgesehen von zahlreichen Wissenshaftern kennen ihn zahlreiche Reisende, mindestens aus der Ostschweiz; nicht zuletzt diesem Umstand verdankt er, daß er schon als interessanteste Straße der Schweiz bezeichnet wurde. Trotzdem ist V. Binggeli sehr zu dan- 